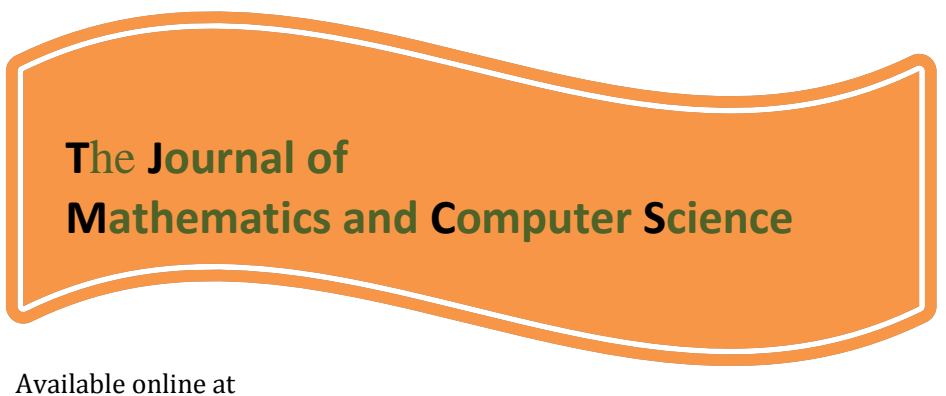

http://www.TJMCS.com

The Journal of Mathematics and Computer Science Vol .3 No.2 (2011) 176 - 178

\title{
On the determination of asymptotic formula of the nodal points for differential pencils with separated boundary conditions
}

\author{
A. Dabbaghian' ${ }^{1}$ and Sh. Akbarpoor ${ }^{2}$ \\ 1'Islamic Azad University, Neka Branch, Neka, Iran. \\ a.dabbaghian@math.com \\ 2Islamic Azad University, Jouybar Branch, Jouybar, Iran. \\ sh.akbarpour@iaujouybar.ac.ir
}

Received: May 2011, Revised: June 2011

Online Publication: July 2011

\begin{abstract}
In this work, we solve the inverse nodal problem for the diffusion operator on a finite interval with separated boundary conditions. We investigation the oscillation of the eigenfunctions and derive an asymptotic formula for the nodal points. Uniqueness theorem is proved, and a constructive procedure for the solution is provided.
\end{abstract}

Keywords: Differential pencils; Eigenvalues; Eigenfunctions; Nodal Points.

\section{Introduction and Preliminaries}

We study inverse nodal problem for the diffusion operator. Inverse nodal problems consist in constructing operators from the given nodes (zeros) of eigenfunctions. Mclaughlin seems to have been the first to consider this sort of inverse problem for the one-dimensional Schrodinger equations on an interval with Dirichlet boundary conditions. In the present paper, we investigate the inverse nodal problem for second-order differential equation with a linear dependence on the spectral parameter(pencils) $L\left(q_{1}(x), h, H\right)$ of the form:

$$
L y(x):=y^{\prime \prime}(x)+\left(\rho^{2}-2 \rho q_{1}(x)-q_{0}(x)\right) y(x)=0, \quad 0<x<1,
$$




$$
U(y):=y^{\prime}(0)-h y(0)=0, \quad V(y):=y^{\prime}(1)+H y(1)=0,
$$

Here $\rho$ is the spectral parameter. Let $q_{0}(x), q_{1}(x)$ are real functions, $h, H$ are real numbers and $q_{0}(x), q_{1}(x) \in W_{1}^{2}[0,1]$.

In [2] the uniqueness of recovering the function $q_{0}(x)-\int_{0}^{1} q_{0}(t) d t$ from the nodal points was studied, provided that $q_{1}(x)$ was known a priori. Such in [1], authors considered the inverse nodal problem for (1.1) with Dirichlet boundary conditions.

In section 2, we obtain the eigenvalues and eigenfunctions corresponding to large modulus eigenvalues. Such calculate an asymptotic of the nodal points.

\section{Computation the nodal points}

Let $\varphi(x, \rho), C(x, \rho), S(x, \rho)$ be the solutions of equation (1.1) under initial conditions $C(0, \rho)=S^{\prime}(0, \rho)=\varphi(0, \rho)=1, C^{\prime}(0, \rho)=S(0, \rho)=0, \varphi^{\prime}(0, \rho)=h$. Then $U(\varphi)=0$. The eigenvalues of L coincide with the zeros of its characteristic function $\Delta(\rho)=-V(\varphi)$. Denote

$$
Q(x)=\int_{0}^{x} q_{1}(t) d t, \quad \int_{0}^{1} q_{1}(t) d t=0 .
$$

Using the standard approach one can establish the asymptotics

$$
S(x, \rho)=\frac{\sin (\rho x-Q(x))}{\rho}+\xi(x, \rho)
$$

where

$$
\xi^{(v)}(x, \rho)=O\left(\frac{1}{\rho^{2-v}} e^{|\tau| x}\right), \quad v=0,1, \quad|\rho| \rightarrow \infty
$$

and

$$
C(x, \rho)=\cos (\rho x-Q(x))+\frac{\sin (\rho x-Q(x))}{2 \rho} \int_{0}^{x} q_{1}(t) d t+\eta(x, \rho)
$$

where

$$
\eta^{(v)}(x, \rho)=O\left(\frac{1}{\rho^{2-v}} e^{|\tau| x}\right), \quad v=0,1, \quad|\rho| \rightarrow \infty
$$

uniformly with respect to $x \in[0,1]$. Since $\varphi(x, \rho)=C(x, \rho)+h S(x, \rho)$, for $|\rho| \rightarrow \infty$ uniformly in $\mathrm{x}$ and using (2.2),(2.3) one has:

$\varphi(x, \rho)=\cos (\rho x-Q(x))+\frac{\sin (\rho x-Q(x))}{\rho}\left(h+\frac{1}{2} \int_{0}^{x} q_{1}(t) d t\right)+O\left(\frac{1}{\rho^{2}} e^{|\tau| x}\right)$.

It follows from (2.4) that for $|\rho| \rightarrow \infty$

$$
\Delta(\rho)=\left(\rho-q_{1}(1)\right) \sin \rho-(h+H) \cos \rho+O\left(\frac{1}{\rho} e^{|\tau|}\right) .
$$

Using (2.5) by the well-known method one has that for $n \rightarrow \infty$, 


$$
\rho_{n}=n \pi+\frac{h+H}{n \pi-q_{1}(1)}+O\left(\frac{1}{n^{2}}\right)
$$

The eigenfunctions of the boundary problem $L$ have the form $y_{n}(x)=\varphi\left(x, \lambda_{n}\right)$. Substituting (2.6) into (2.4) we obtain the following asymptotic formulae for $n \rightarrow \infty$ uniformly in $\mathrm{x}$ :

$$
\begin{aligned}
& y_{n}(x)=\cos (n \pi x-Q(x))-\sin (n \pi x-Q(x)) \frac{h+H}{n \pi-q_{1}(1)}(x) \\
& +\frac{h+\frac{1}{2} Q(x)}{n \pi} \sin (n \pi x-Q(x))+O\left(\frac{1}{n^{2}}\right) \quad 4 c m
\end{aligned}
$$

For the boundary value problem $L$ an analog of Sturm's oscillation theorem is true. More precisely, the eigenfunction $y_{n}(x)$ has exactly $n$ zeros inside the interval $(0,1)$.

Namely, $0<x_{n}^{1}<\ldots<x_{n}^{n}<1$. Denote $\alpha_{n}^{j}=\frac{\left(j-\frac{1}{2}\right)}{n}$. Taking (2.7) into account, we obtain the following asymptotic formulae for nodal points as $n \rightarrow \infty$ uniformly in $\mathrm{j}$ :

$$
x_{n}^{j}=\alpha_{n}^{j}+\frac{1}{n \pi}\left[-\frac{h+H}{n \pi-q_{1}(1)} x_{n}^{j}+\frac{h+\frac{1}{2} Q\left(x_{n}^{j}\right)}{n \pi}+Q\left(x_{n}^{j}\right)\right]+O\left(\frac{1}{n^{3}}\right) .
$$

Using this formulae, we arrive at the following assertion.

Theorem 2.1. Fix $x \in[0,1]$. Choose $j_{n}$ such that $x_{n}^{j_{n}} \rightarrow x$ as $n \rightarrow \infty$. Then there exists finite limits and the corresponding equalities hold:

$$
\begin{aligned}
& Q(x)=\pi \lim _{n \rightarrow \infty}\left[n x_{n}^{j_{n}}-\left(j_{n}-\frac{1}{2}\right)\right] \\
& f(x)=\pi \lim _{n \rightarrow \infty}\left[n\left(\pi\left(n x_{n}^{j_{n}}-\left(j_{n}-\frac{1}{2}\right)\right)-Q\left(x_{n}^{j_{n}}\right)\right)\right]
\end{aligned}
$$

and

$$
f(x)=-(h+H) x+h+\frac{1}{2} Q(x)
$$

Acknowledgments. This research was supported by Islamic Azad University, Neka Branch.

\section{References}

[1] Buterin, S. A., Chung Tsun Shieh, Inverse nodal problem for differential pencils, Appl. Math. Lett.,22(8)(2009)

[2] Koyunbakan, H., A new inverse problem for the diffusion operator, Appl. Math. Lett.,19(2006) 995-999. 